\title{
Modelo de negocios para la gestión de la cadena de suministro: una revisión y análisis bibliométrico
}

Business model for supply chain management:

a review and bibliometric analysis

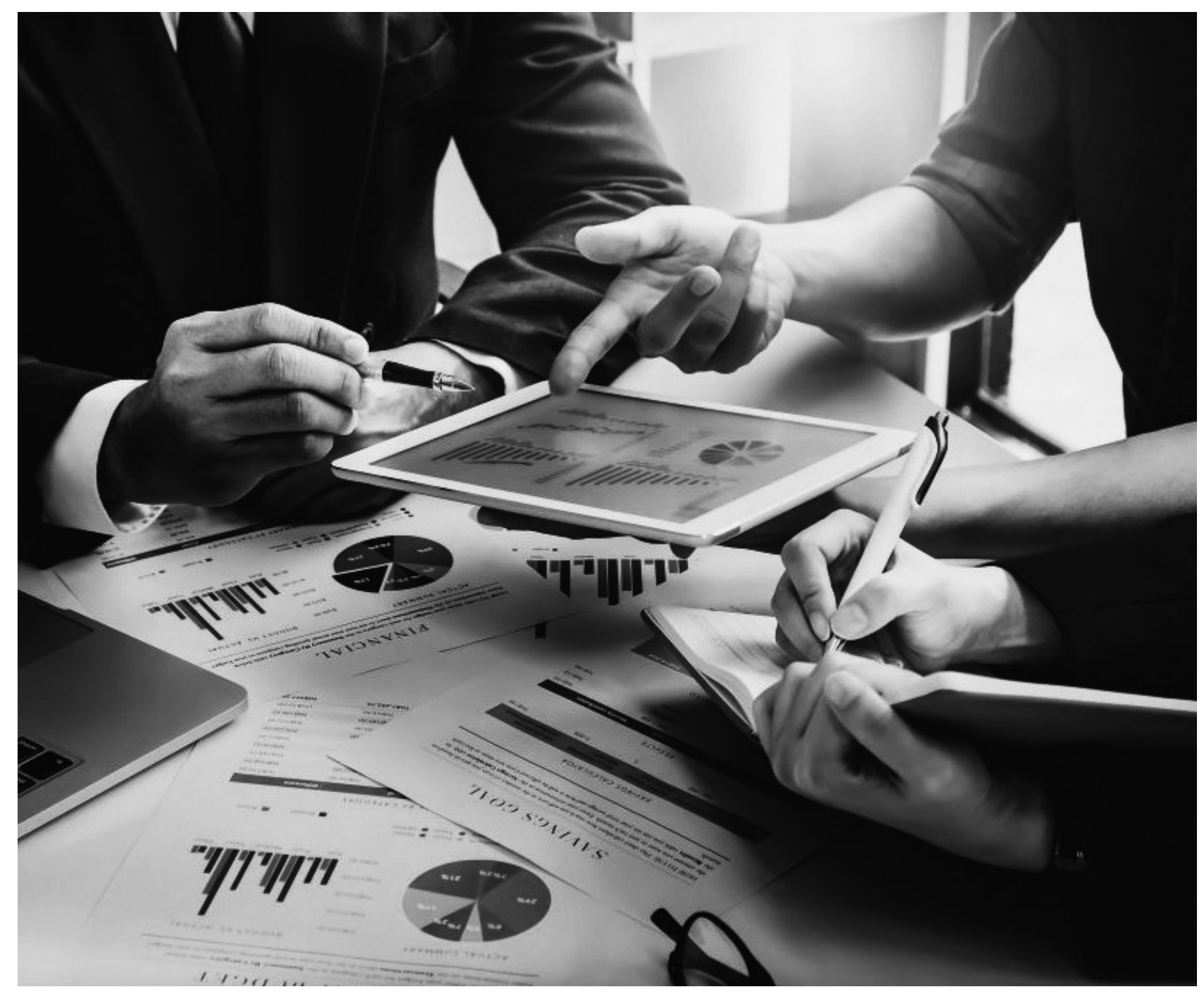




\title{
Modelo de negocios para la gestión de la cadena de suministro: una revisión y análisis bibliométrico ${ }^{1}$
}

\author{
Business model for supply chain management: \\ a review and bibliometric analysis
}

\author{
Sara Delgado Gil ${ }^{2}$, Jairo Gonzalez Bueno ${ }^{3}$, Jairo Núñez Rodríguez ${ }^{4}$
}

Artículo recibido en julio de 2017; artículo aceptado en septiembre de 2017.

\begin{abstract}
Este artículo puede compartirse bajo la Licencia Creative Commons Atribución-No Comercial-Compartir Igual 2.0 Genérica
y se referencia usando el siguiente formato: Gil, S, Gonzalez, J. \& Nuñez, J. (2018). Modelo de negocios para la gestión

de la cadena de suministro: una revisión y análisis bibliométrico. I+D Revista de Investigaciones, 11(1), 38-55.
\end{abstract}

DOI: https://doi.org/10.33304/revinv.v11n1-2018004

\begin{abstract}
Resumen
El campo de los modelos de negocio para gestionar las cadenas de suministros en las organizaciones ha ido evolucionando rápidamente y ganado popularidad e importancia en la comunidad investigativa. Este artículo busca identificar y caracterizar por medio de una revisión de la literatura los modelos de negocios para la gestión de las cadenas de suministro, con el propósito de ofrecer una visión profunda en este campo. Mediante un análisis bibliométrico, la investigación evaluó 665 artículos en torno a modelos de negocios publicados en los últimos diez años, identificando los principales autores, organizaciones y temas claves de investigación.
\end{abstract}

Palabras clave: modelos de negocio, cadenas de suministro, análisis bibliométrico.

\begin{abstract}
The field of business models for managing supply chains in organizations has been evolving rapidly and gaining popularity and importance in the research community. This article seeks to identify and characterize by means of a review of the literature available business models for the management of supply chains with the purpose of providing a deep vision in this field. Through a bibliometric analysis, the research evaluated 665 articles related to business models published in the last ten years, identifying the main authors, organizations and key research topics.
\end{abstract}

Keywords: business model, suply chain, bibliometric analysis.

1. El tipo de artículo es de revisión documental de enfoque cualitativo, resultado de la tesis de pregrado (en curso), perteneciente al área de Logística y Cadena de suministro, desarrollado en el grupo de investigación Geetic -Investigación en Empresa, TIC y Educación de la Universidad Pontificia Bolivariana.

2. Estudiante Ingeniería Industrial, Universidad Pontificia Bolivariana, Bucaramanga, Colombia: Campus Universitario Kilómetro 7 vía a Piedecuesta. sara.delgado@upb.edu.co.

3. Ingeniero financiero y contador público, Universidad Autónoma de Bucaramanga. Magister en Gerencia de Negocios, Universidad Industrial de Santander. Candidato a doctor en Administración y Dirección de Empresas, Universidad Politécnica de Valencia, España. Docente investigador Grupo de Investigación en Administración - GIA. Universidad Pontificia Bolivariana, Bucaramanga, Colombia: Campus Universitario Kilómetro 7 vía a Piedecuesta. ORCID ID: https://orcid.org/0000-0002-5896-094X. Correo electrónico institucional: jairoa.gonzalez@upb.edu.co.

4. Ingeniero industrial, Universidad Pontificia Bolivariana. Candidato a doctor en Ingeniería y Producción Industrial, Universidad Politécnica de Valencia, España. Docente investigador - Grupo Geetic -Investigación en Empresa,TIC y Educación. Universidad Pontificia Bolivariana, Bucaramanga, Colombia: Campus Universitario Kilómetro 7 vía a Piedecuesta. ORCID ID: https://orcid.org/0000-0002-4301-9081 Correo electrónico institucional: jairo.nunez@upb.edu.co. 


\section{Introducción}

La innovación en los modelos de negocios de las empresas abre la oportunidad no solo de transformar la propuesta de valor, la arquitectura de valor o el modelo de sus ingresos, sino también de repensar sus sistemas de valores humanos y así crear negocios que puedan superar las expectativas de los consumidores. Cada vez más se espera de las empresas que sean estrictamente responsables con sus operaciones comerciales y su ética empresarial; de otra parte, las limitaciones ambientales también son cada vez mayores, por lo tanto, la línea de responsabilidad esperada se extiende a lo largo de todos los eslabones de la cadena de suministro. Por estas razones, las empresas deben formar un sistema de valor definido en sus modelos de negocios (Pinto \& Quintero, 2017).

Gran cantidad de literatura explora las razones por las cuales las organizaciones deben tener su sistema de valor definido y cómo implementarlo, aun así, no en todas se implementan estos sistemas de manera adecuada y no logran cumplir con los objetivos establecidos. En este sentido, es evidente la necesidad de integrar la administración de la cadena de suministro con los modelos de negocio de las empresas. Dada la variedad de estos campos existe una necesidad tangible de desarrollar una comprensión mejor y más centrada acerca de la manera como se definen, interpretan, investigan y se llevan a la práctica.

En este sentido, se hará una revisión bibliográfica de los modelos de negocio para la gestión de las cadenas de suministros; específicamente, la investigación busca abordar las siguientes preguntas: ¿cuáles han sido los aportes sobre los diferentes modelos de negocio para la gestión la cadena de suministros desarrollados en los últimos diez años? ¿Cuál es el desarrollo alcanzado frente a los diferentes modelos de negocio para la gestión de las cadenas de suministro?

Abordando estas preguntas, mediante esta revisión y la aplicación de indicadores bibliométricos, se pretende comprender el contexto de la temática para así proveer a los lectores un material de fácil entendimiento que sirva como parámetro base para futuras investigaciones.

\section{Método}

\section{Tipo de estudio}

Se trata de una investigación con un enfoque cualitativo que buscar agrupar estudios relacionados con modelos de negocio y cadena de suministros, con el fin de generar conclusiones a partir de un análisis descriptivo. El tipo de estudio es no experimental, aunque mide y evalúa aspectos de documentación científica, clasificando y valorando la información que se puede extraer de los artículos científicos, y es de tipo longitudinal por que revisa la trazabilidad de la literatura de los últimos diez años de publicación.

\section{Participantes}

Se consideran como participantes de análisis los artículos científicos encontrados en bases de datos, se define como la expresión que designa un informe original de investigación (Day, 2005), los cuales son arbitrados por pares académicos si quieren estar publicados en revistas científicas de prestigio. Las revistas están inscritas en bases de datos que tienen por objetivo recopilar un conjunto de revistas asociadas a áreas de conocimiento.

\section{Materiales e instrumentos}

Los instrumentos utilizados para el desarrollo de la investigación fueron el software Vantage Point, una herramienta de minería de texto que ayuda a entender rápidamente grandes volúmenes de información (The Vantage Point, 2012) y permite hacer análisis de coocurrencia, mapas/ redes, limpieza de datos entre otros; y el VOSviewer, que permite construir y visualizar redes bibliométricas (Leiden University, 2010).

\section{Procedimiento}

El procedimiento se divide en dos fases:

\section{Fase I. Necesidad de información.}

Revisión de la literatura.

Identificación de los términos clave de búsqueda.

Refinamiento de la búsqueda.

\section{Fase II. Análisis bibliométrico.}

Tendencia de publicación.

Ubicación espacial.

Artículos de mayor relevancia.

Análisis de palabras clave.

Propuesta de grupos temáticos.

\section{Resultados}

\section{Fase I. Necesidad de información}

Revisión de la literatura. Blanchard (2010) define la cadena de suministro como la secuencia de eventos que cubren el ciclo de vida entero de un producto o servicio desde que es concebido hasta que es consumido. La 
cadena de suministros no está limitada a empresas manufactureras, sino que se ha ampliado para incluir tanto "productos tangibles" como "servicios intangibles" que llegan al consumidor, y requieren a su vez insumos de productos y servicios (Ayers, 2006).

Mentzer et al. (2001) definen la administración de la cadena de suministros como la coordinación sistemática y estratégica de las funciones tradicionales del negocio, a través de estas funciones empresariales dentro de una compañía en particular, y a través de las empresas que participan en la cadena de suministros con el fin de mejorar el desempeño a largo plazo de las empresas individuales y de la cadena de suministros como un todo. Esta es una tarea que una empresa no puede hacer por sí misma o que si la hiciera sus costos se verían bastante incrementados. Por esta razón, Ballou (2004) afirma que en general una sola empresa no es capaz de controlar todo su canal de flujo de producto, desde la fuente de la materia prima hasta los puntos de consumo final.

Para propósitos prácticos, la logística de los negocios para una empresa individual tiene un alcance más limitado.

Normalmente, el máximo control gerencial que puede esperarse acaba en el suministro físico inmediato y en los canales físicos de distribución, esto debido a las semejanzas en las actividades entre los dos canales, el suministro físico (conocido como administración de materiales) y la distribución física que comprende aquellas actividades funcionales (transporte, control de inventarios, etc.) que están integradas en la logística de los negocios. La dirección de la logística de los negocios se conoce ahora popularmente como dirección de la cadena de suministros. Se usan otros términos, como redes de valor, corrientes de valor y logística ágil para describir un alcance y un propósito parecido (Ballou, 2004).

Para la cadena de suministro es importante apostar por un modelo de negocios cohesionado y de alto rendimiento basado en la unificación de funciones y procesos dentro de las otras empresas. Además de las actividades logísticas también incluye la gestión de las operaciones de fabricación y dirige la coordinación de procesos y actividades a través de las tecnologías de la información y comunicación TIC, las finanzas, el área de marketing y otras.

Según Al-Debei, El-Haddadeh y Avison (2008) un modelo de negocios es una representación abstracta de una organización, ya sea de manera textual o gráfica, de todos los conceptos relacionados, acuerdos financieros, así como del portafolio central de productos o servicios que la organización ofrece y ofrecerá con base en las acciones necesarias para alcanzar sus metas y objetivos estratégicos.
Algunos autores definen el modelo de negocio de diferentes maneras; en la Tabla 1 se presenta una clasificación de las definiciones seleccionadas para el concepto de modelo de negocios.

Según estos conceptos se puede inferir que, aunque el término de modelo de negocios es relativamente nuevo, es muy usado para referirse a la representación abstracta de una organización; asimismo, se puede afirmar que no existe una definición dominante para este término.

La logística surge en varios casos como el eje central sobre el cual gira el modelo de negocio, dando lugar a lo que puede denominarse como "negocios basados en la logística" (Giannice, 2013). Es decir, que la logística no surge dentro de los modelos de negocio solo como un componente más, el cual actúa funcionalmente respecto de otras variables de la estructura del negocio, sino que en muchos casos se constituye en el elemento central, alrededor del cual se conjugan las demás variables que apuntalan la competencia logística.

Para que una empresa pueda adoptar un modelo de negocio para gestionar su cadena de suministros, es importante conocer su nivel de madurez. Según Röglinger, Pöppelbuß y Becker (2012) basados en el supuesto de patrones predecibles de evolución y cambio organizacional, los modelos de madurez típicamente representan teorías sobre cómo las capacidades de una organización evolucionan de una manera fase por etapa a lo largo de la trayectoria anticipada, deseada o lógica. En consecuencia, también se denominan modelos de etapas de crecimiento, modelos de escenario o teorías de etapas.

Identificación de los términos clave de búsqueda. Se agrupan dos grandes temas; por un lado, modelos de negocio y por el otro, cadena de suministro. Usando las palabras clave se elaboró la ecuación de búsqueda de la revisión bibliográfica como se muestra en la Figura 1. Adicionalmente, la búsqueda realizada con la ecuación de búsqueda se hizo usando los filtros de búsqueda en título, palabras clave y resumen.

Por ser la mayor base de datos de resúmenes y citas de la literatura revisada por pares (Elsevier, 2017), se recogieron los documentos que utiliza Scopus, a la que la Universidad Politécnica de Valencia, en España, describe como La base de datos producida por Elsevier que engloba la mayor colección a nivel mundial de resúmenes, actas de conferencias, webs científicas, referencias bibliográficas e índices de más de 15000 revistas con peer-review de ciencias, tecnología, medicina y ciencias sociales. Química, Física, Matemáticas e Ingeniería Ciencias de la Vida y de la Salud, Ciencias Sociales, Psicología y Económicas. 
Tabla 1

\section{Definiciones académicas para el concepto de modelo de negocios}

\begin{tabular}{|c|c|}
\hline Autor & Definición \\
\hline Amit y Zott (2001) & $\begin{array}{l}\text { Un modelo de negocio representa el diseño del contenido, su estructura y la manera de crear } \\
\text { valor a través de la explotación de nuevas oportunidades de negocios. }\end{array}$ \\
\hline Dubosson-Torbay, & La arquitectura de la organización y su red de socios para crear, comercializar y entregar valor \\
\hline Osterwalder y Pigneur (2002) & $\begin{array}{l}\text { en relación de uno o varios segmentos de clientes, con el fin de generar ingresos rentables y } \\
\text { sostenibles }\end{array}$ \\
\hline Stähler (2002) & $\begin{array}{l}\text { Un modelo de un negocio existente o un futuro negocio planificado. Un modelo es siempre una } \\
\text { simplificación de la compleja realidad. Ayuda a comprender los fundamentos de una empresa o } \\
\text { a planear cómo debe ser un negocio futuro. }\end{array}$ \\
\hline Magretta (2002) & $\begin{array}{l}\text { El modelo de negocio cuenta una historia lógica que explica quiénes son los clientes, lo que } \\
\text { valoran, y cómo el empresario hará dinero a partir de los clientes a la vez que les entrega valor. }\end{array}$ \\
\hline Camponovo y Pigneur (2003) & $\begin{array}{l}\text { Una conceptualización detallada de la estrategia de una empresa en un nivel abstracto, que sirve } \\
\text { de base para la implementación de procesos de negocio. }\end{array}$ \\
\hline $\begin{array}{l}\text { Seong Leem, Sik Suh } \\
\text { y Seong Kim (2004) }\end{array}$ & $\begin{array}{l}\text { Un conjunto de estrategias para el establecimiento y gestión de empresas, modelo de ingresos, } \\
\text { procesos de negocios de alto nivel y alianzas. }\end{array}$ \\
\hline Rajala y Westerlund (2005) & $\begin{array}{l}\text { Las formas de crear valor para los clientes y la forma en que el negocio se convierte en mercado } \\
\text { de oportunidades de beneficio a través de conjuntos de actores, actividades y colaboradores. }\end{array}$ \\
\hline $\begin{array}{l}\text { Osterwalder, Pigneur } \\
\text { y Tucci (2005) }\end{array}$ & $\begin{array}{l}\text { Un modelo de negocio es una herramienta conceptual que contiene un conjunto de elementos } \\
\text { relacionados y permite expresar la lógica de negocio de una empresa específica. Es una } \\
\text { descripción del valor que una empresa ofrece a uno o varios segmentos de clientes, de la } \\
\text { arquitectura de la empresa y de su red de socios para crear, comercializar y entregar este capital } \\
\text { de relación de valor, para generar rentabilidad y sostenibilidad. }\end{array}$ \\
\hline Andersson, et al. (2006) & $\begin{array}{l}\text { Los modelos de negocio se crean para aclarar quiénes son los actores del negocio que están en } \\
\text { un caso de negocios y cómo hacer explícitas sus relaciones. Las relaciones en un modelo de } \\
\text { negocio se formulan en términos de valores intercambiados entre los actores. }\end{array}$ \\
\hline Kallio, Tinnilä, y Tseng, (2006) & $\begin{array}{l}\text { Los medios por los cuales una empresa es capaz de crear valor coordinando el flujo de } \\
\text { información, bienes y servicios entre los distintos participantes de la industria cuando esta entra } \\
\text { en contacto con clientes, socios dentro de la cadena de valor, con sus competidores y el } \\
\text { gobierno. }\end{array}$ \\
\hline
\end{tabular}

Fuente: Autores.

El resultado de la aplicación de búsqueda permite realizar un análisis bibliométrico que contribuye a la obtención de estudios más precisos que permiten estudiar variables como títulos de los artículos que han sido publicados, autores más productivos, contenidos relevantes sobre los temas que han tratado los documentos, ubicación espacial, centros o instituciones que han intervenido en las publicaciones y año de publicación de los trabajos.
Refinamiento de la búsqueda. El análisis bibliométrico se centró con una ventana de tiempo para los artículos científicos de los últimos diez años de publicación, en idioma inglés; se excluyeron los documentos con enfoque diferente al tema de investigación.

Para perfeccionar los resultados de búsqueda se utilizó el software VantagePoint y el Voswiver; la búsqueda arrojó 665

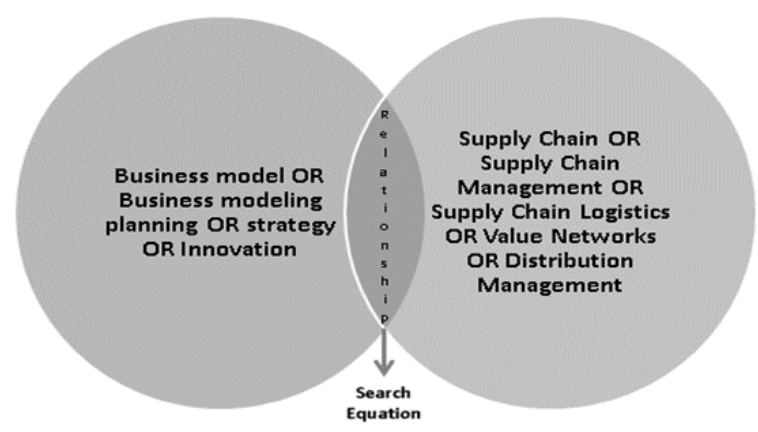

Figura 1.Diagrama de Venn. Ecuación de búsqueda. Fuente: Autores. 
artículos que fueron exportados en formato CSV, el cual contiene la información relacionada con cada documento.

Labúsqueda serefinósegúnel criteriodelosinvestigadores, considerando el título, el resumen y las palabras clave, así como la relación con el tema central:"modelos de negocio para gestionar la cadena de suministro de las empresas". De los 665 documentos se seleccionaron 83 debido a su pertinencia con el tema de estudio.

Los artículos excluidos -porque se desviaban del tema central-en su mayoría trataban de modelos de optimización para las cadenas de suministro, modelos matemáticos, estrategias de integración para proveedores, investigaciones para incrementar la efectividad de las partes interesadas en las cadenas de suministro y comparación entre diferentes industrias.

\section{Fase II. Análisis bibliométrico}

Tendencia de publicación. La Figura 2 evidencia la distribución por años de publicaciones relacionadas con la base de estudio; se observa que la mayor cantidad de publicaciones se hicieron en los años 2009 y 2015, y a partir del 2009 aumenta el número de publicaciones.

\section{Publicaciones por años}

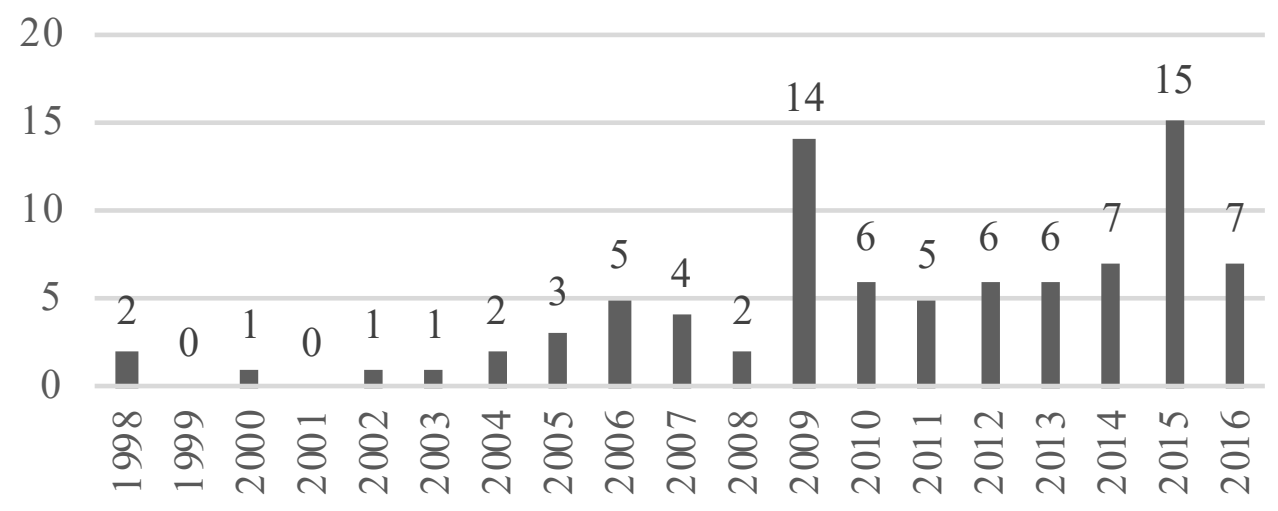

Figura 2. Trazabilidad de publicación. Fuente: software VantagePoint.

Entre los temas tratados en el año 2009, Tian-Min (2009) presenta un modelo de negocios de red global basado en una plataforma de colaboración E-business logrando un modelo Business to Business (B2B) de comercio electrónico. Por su parte, Tan, Ahmed y Sundaram (2009) exploran la aplicación de los principios de sostenibilidad en el contexto de la gestión de almacenamiento y distribución. Por otro lado, Vurro, Russo y Perrini (2009), en un intento de predecir la variedad en los modelos de gobernabilidad relacionados con la sostenibilidad a lo largo de la cadena de valor, proponen un modelo de negocios teórico que identifica y enmarca cuatro modelos de gobernabilidad sostenible de la cadena de suministro.

En 2015 también es notoria la cantidad de publicaciones que desarrollan estas temáticas, como una propuesta de red logística hacia adelante/atrás y un modelo de planificación de rutas para las empresas de comercio electrónico de alimentos frescos (Siyuan, Xu, Liang \& Guo, 2015); Prause (2015) discute la posición industrial global de Europa, representada por la industria 4.0, con el objetivo de aplicar la producción inteligente y la logística 4.0, la cual toca toda la cadena de suministro que comprende el diseño, el desarrollo de productos y la gestión de operaciones (Díaz, 2014).

Ubicación espacial. En la Figura 3 se evidencia el número artículos por países, de mayor a menor. Es notorio que la mayoría de los artículos fueron producidos en China, 18 de 83; Reino Unido, 7 de 83, en Estados Unidos, 10 de 83. Los 50 artículos restantes se encuentran en diferentes países alrededor del mundo.

Artículos de mayor relevancia. La relevancia se refiere al impacto de los artículos basados en el número de veces que son citados; se destacan los cinco artículos más importantes, como se muestra en la Tabla 2.

Encabeza la lista el artículo "Reengineering the order fulfillment process in supply chain networks", con 122 citaciones, elaborado por Furen Lin de la Universidad Nacional Tsing 


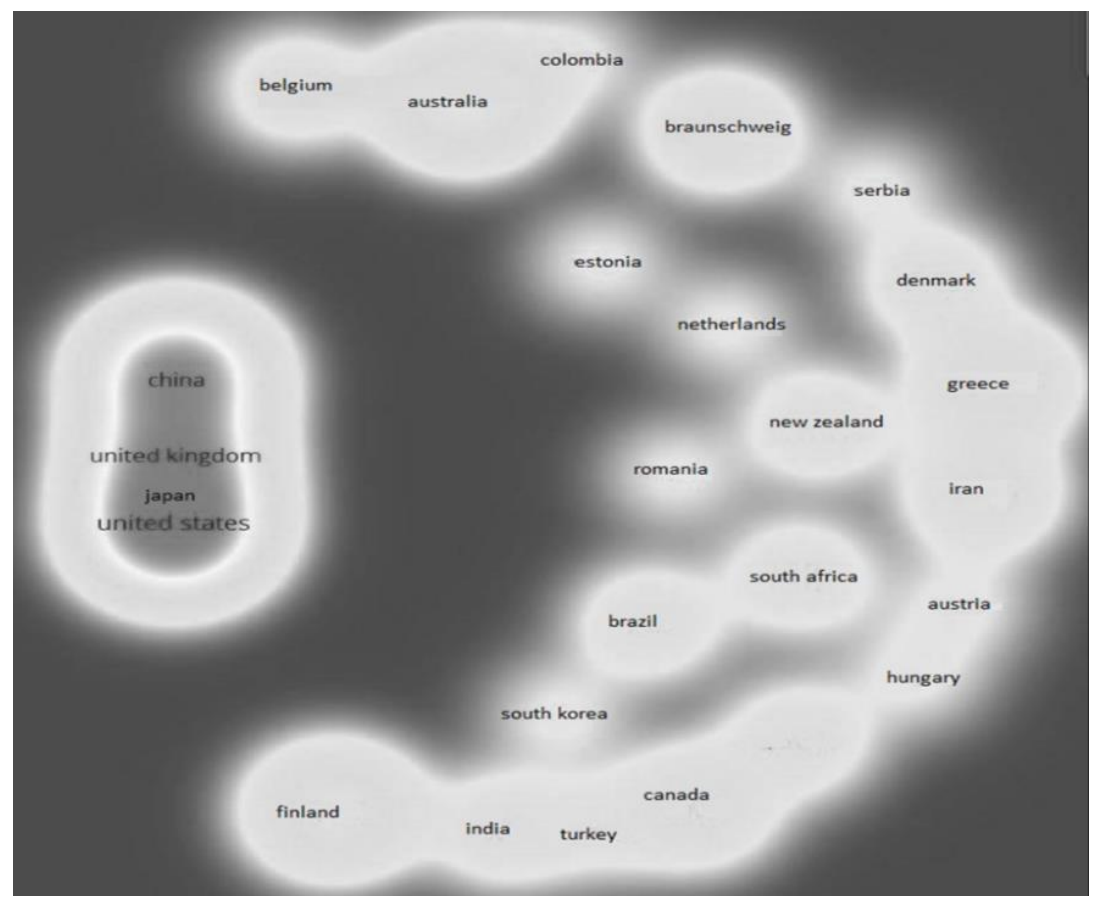

Figura 3. Ubicación espacial. Fuente: software Vosviewer.

Hua, Instituto de Ciencia de Servicios, Hsinchu, Taiwán y Michael Shaw de la Universidad de Illinois, Estados Unidos.

En segundo lugar, está el artículo "Virtual e-chain (VeC) model for supply chain collaboration", con 93 citaciones, escrito por Vicky Manthou y Maro Vlachopoulou de Panepistimion Makedonias, del Departamento de Informática Aplicada, Tesalónica, Grecia y Dimitris Folinas de TEI-KM, Departamento de Logística, Grecia.
En tercer lugar, se ubica el artículo "A contingency model for creating value from RFID supply chain network projects in logistics and manufacturing environments", con 64 citaciones, escrito por Samuel Fosso, de la Escuela de Negocios Toulouse, Toulouse, Francia, y Akemi Chatfield de la Escuela de Tecnología de la Información y Ciencias de la Computación de la Universidad de Wollongong, Wollongong, Australia.

\section{Tabla 2}

Top 5 de los artículos más citados

\begin{tabular}{lc}
\hline \multicolumn{1}{c}{ Título } & Citas \\
\hline $\begin{array}{l}\text { Reengineering the order fulfillment processin supply chain networks } \\
\text { Virtual e-chain (VeC) model for supply chain collaboration. }\end{array}$ & 122 \\
$\begin{array}{l}\text { A contingency model for creating value from RFID supply chain network projects } \\
\text { in logistics and manufacturing environments. }\end{array}$ & 93 \\
$\begin{array}{l}\text { A decision-making model for lean, agile, resilient and green supply chain } \\
\text { management. }\end{array}$ & 42 \\
$\begin{array}{l}\text { Shaping sustainable value chains: network determinants of supply chain } \\
\text { governance models }\end{array}$ & 34 \\
\hline
\end{tabular}

Fuente: Autores. 
Seguidamente se encuentra el artículo "A decision-making model for lean, agile, resilient and green supply chain management", con 42 citaciones, producido por Izunildo Cabral,del DepartamentodelngenieríaMecánicaelndustrial de la Universidad de Nova de Lisboa, Lisboa, Portugal, y Antonio Grilo, de la Facultad de Ciencias y Tecnología de la Universidad Nueva de Lisboa, Caparica, Portugal, y Virgilio Cruz-Machado, del Unidemi (Departamento de Ingeniería Mecánica e Industrial), Facultad de Ciencias y Tecnología de la Universidad Nueva de Lisboa, Caparica, Portugal.

En último lugar está el artículo "Shaping sustainable value chains: network determinants of supply chain governance models", con 34 citaciones, desarrollado por Clodia Vurro, del Departamento de Gestión y Tecnología de la Universidad L. Bocconi, Milán, Italia; Angeloantonio Russo Departamento de Gestión de la LUM Universidad, Italia, y Francesco Perrini, de SIF Cátedra de Emprendimiento Social y Filantropía de la Universidad Bocconi, Milán, Italia.

Finalmente, el artículo"The potential of RFID in warehousing activities in a retail industry supply Chain", con 30 citaciones, elaborado por Louis André Lefevbre y Elisabeth Lefevbre de la Escuela Politécnica de Montreal, Departamento de Matemáticas e Ingeniería Industrial, Montreal, Canadá; Ygal Bendavid del Departamento de Gestión y Tecnología de la Universidad de Quebec, Montreal, Montreal, Canadá; Samuel Fosso de Toulouse Business School, Toulouse, Francia y Harold Boeck, del Departamento de Marketing de la Universidad de Quebec, Montreal, Canadá.

Análisis de palabras clave. Para realizar una búsqueda válida y confiable son necesarias las palabras claves. En la Figura 4 se observa que las palabras más utilizadas en los resultados arrojados fueron supply chains y supply chain management, lo que indica que la mayoría de los artículos están relacionados con el objeto de estudio; adicionalmente, se pueden observar otras palabras relacionadas como electronic commerce, sustainable development, internet, manufacture, entre otras. Esto quiere decir que pueden ser subtemas que tienen relación con el análisis de los modelos de negocio.

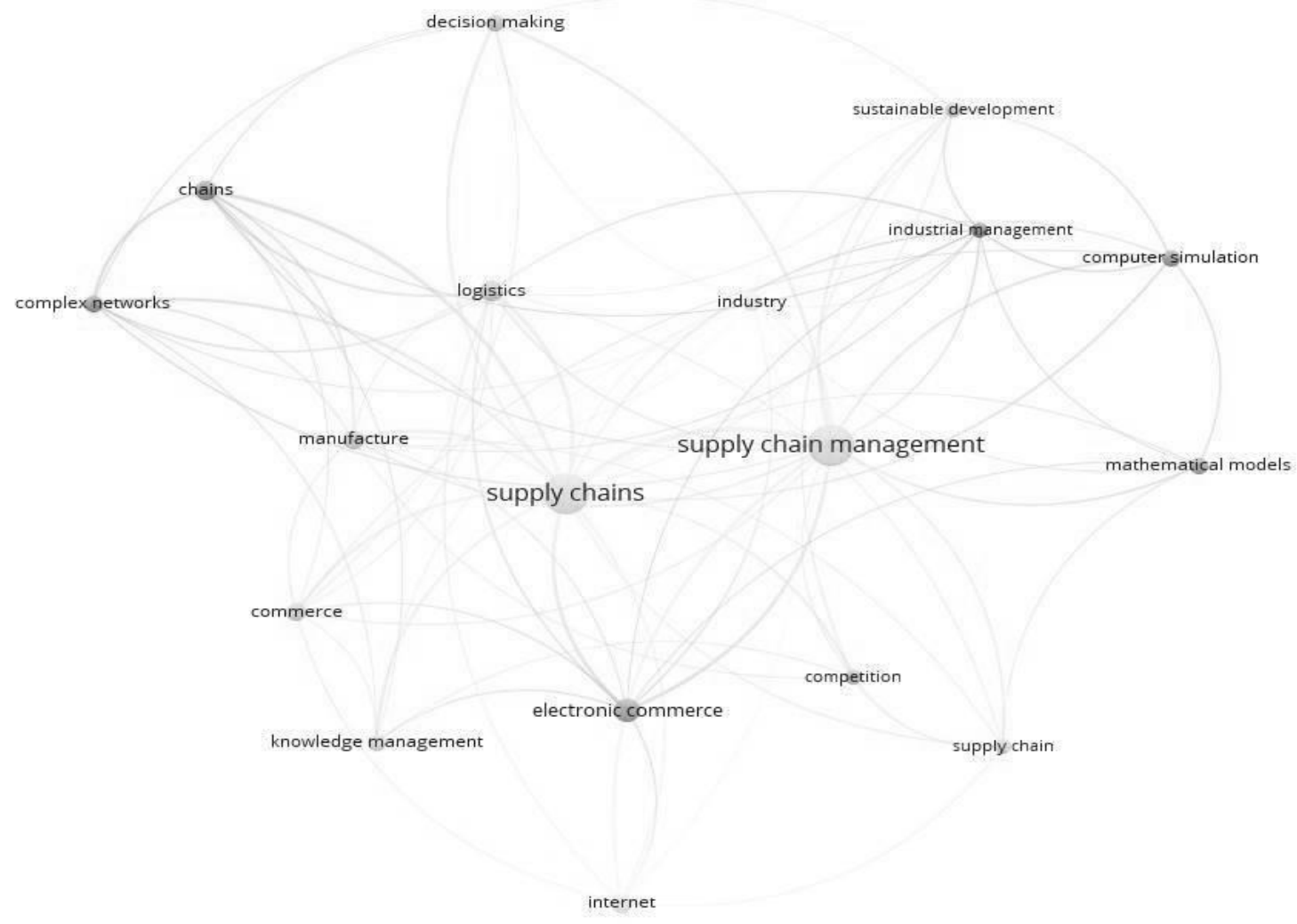

Figura 4. Relación de palabras clave. Fuente: software Vosviwer. 
Grupos temáticos. Habiendoanalizadosistemáticamente los resultados encontrados, se clasificaron los artículos para identificar grupos temáticos. Se consideraron seis grupos de modelos de negocios encontrados en los documentos, algunos de los cuales se hallan en más de un grupo debido a que contienen más de un tema.

La Tabla 3 presenta los temas identificados y enumera todos los documentos relacionados; la referencia asignada a cada uno de los temas se encuentra en el Apéndice A.

Adicionalmente, el gráfico nos muestra la utilización de los términos a través del tiempo, destacándose que a mediados del año 2012 se comienza la relación con manufacture, commerce, logistics, decision making y sustainable Development.

Tabla 3

Propuesta de agrupación de temática

\begin{tabular}{ll}
\hline \multicolumn{1}{c}{ Eje temático } & \multicolumn{1}{c}{ Título (apéndice A) } \\
\hline Modelos genéricos & $10,14,38,54,61,78,82$ \\
Cadenas de suministro & $1,2,7,8,11,12,14,16,19,20,23,28,30,31,32,33,39,52,58,59,60,62,69,70,73,75,77,79,80$ \\
Modelos teóricos & $3,5,8,17,21,26,29,37,42,43,45,47,49,50,57,58,65,67,69,71,76$ \\
Modelos prácticos & $4,6,25,27,34,35,36,40,41,46,52,53,55,56,63,64,66,68,81,83$ \\
E-Commerce & $6,7,22,24,32,41,48,72,75$, \\
B2B & $9,15,32,44,74$ \\
\hline
\end{tabular}

Fuente: Autores.

Modelos genéricos. Se propone el grupo de los modelos genéricos debido a que hacen referencia a las tareas tradicionales para la gestión de las cadenas de suministro, por lo que se tratan temas transversales en los cuales es indiferente el tipo de negocio. En la clasificación de los resultados de la búsqueda el grupo está representado por siete artículos. De acuerdo con los resultados de la revisión de la literatura, Panou (2015) afirma que mientras que las redes de valor de servicio (SVN) buscan continuamente nuevos vínculos entre los socios para crear valor, los proveedores de servicios se ven obligados a reestructurar sus modelos de negocio para que se vuelvan más centrados en el cliente. Una consecuencia de esto ha sido un cambio del enfoque tradicional en la producción a un enfoque en la construcción de relaciones con clientes y proveedores. Para abordar este problema el autor ha desarrollado un modelo personalizado de valor de vida útil del cliente (LTV) que cumpla con los requisitos de negocio de SVN. Con el objetivo de ayudar a los socios de servicio en la evaluación de la viabilidad de sus redes se fusiona el enfoque LTV con el concepto de modelo de negocio "freemium" y se aplica a una cadena de suministro SVN.

La aplicación del modelo consta de cinco pasos: adquisición de usuarios, conversión gratuita, retención de usuarios, análisis de flujo de efectivo y cálculo de LTV. La adquisición de usuarios se basa en tres escenarios: el escenario optimista, el tendencial y el pesimista, que se construyen en torno al grado de penetración en el mercado de los servicios SVN y las decisiones de inversión privada para captar las fuerzas de colaboración y emprendedoras.
Otro reconocimiento significativo en la literatura revisada es un modelo de simulación genérico que se utiliza para investigar el impacto del desempeño de la cadena de suministro respecto a la variedad de productos, en combinación con el plazo de suministro y la incertidumbre de la demanda en un entorno internacional. El estudio muestra que el aumento del nivel de variedad de productos tiene un impacto perjudicial en el desempeño de la cadena de suministro.

Con la incertidumbre de la demanda, los niveles altos de la variedad dan lugar a tiempos de flujo mucho más largos y a un inventario mucho más alto. El impacto es mayor cuando la variedad implica materiales críticos que se requieren a principios del proceso de producción y que implican largos tiempos de preparación (Er \& MacCarthy, 2006).

Cadenas de suministro. El grupo de las cadenas de suministro (CS) se seleccionó debido a que son modelos de negocio que buscan el máximo funcionamiento de la CS abarcando todos los eslabones de esta, aplicados en determinadas empresas de diversas industrias. En esta revisión, dicho grupo está representado por 29 artículos.

La gestión de la cadena de suministro (SCM) tiene como objetivo integrar eficientemente los proveedores, fabricantes, almacenes y minoristas para asegurar que la mercancía se produce y se distribuye en las cantidades adecuadas, en las ubicaciones correctas y en el momento óptimo, a la vez que se satisfacen las necesidades de los clientes (Hsieh \& Hung, 2009). En la literatura revisada se 
pudo observar que las relaciones entre las cadenas de suministro son una vía clave que influye en el rendimiento de las empresas, por lo tanto, se deben dinamizar todos los procesos que la componen. Lin \& Shaw (1998) realizaron un estudio en el cual identifican varios tipos principales de estructuras de red de la cadena de suministro ( $\mathrm{SCN}$ ) y abordaron los temas de cumplimiento de pedidos (OFP) basados en ellos. El objetivo de la reingeniería del OFP es agilizar el proceso en términos de eficiencia, flexibilidad, robustez y adaptabilidad, en el que se aplicaron estrategias como (1) coordinación de políticas de gestión de la demanda, (2) estrategias de intercambio de información, (3) sincronización de material y disponibilidad de capacidad, (4) asignación dinámica de recursos y (5) combinación de diversas estrategias.

Los resultados permitieron identificar los principales efectos de diversas estrategias en el desempeño de los OFP, a partir de lo cual nace la idea de utilizar estas estrategias en diferentes $\mathrm{SCN}$ que ayudan a reestructurar el OFP en SCN de empresas manufactureras.

La literatura revisada también muestra que otra manera de fortalecer las cadenas de suministros de una empresa es que las compañías independientes comiencen a trabajar juntas basadas en valores compartidos y un objetivo común, en hacer negocios para explotar conjuntamente una oportunidad de negocio particular. La especificación del modelo de negocio de cada socio, su coordinación organizativa y técnica y los criterios adecuados para los acuerdos entre ellos pueden resolver el problema de combinar las diferentes competencias de los socios (Manthou, Vlachopoulou \& Folinas, 2004). Estos investigadores desarrollaron un modelo relacionado también con el grupo E-commerce (se especifica más adelante), que presenta un marco de colaboración de la cadena de suministro en un entorno virtual para clasificar las funciones de los asociados, identificar las capacidades clave para estructurar cada relación de colaboración y evaluar la disponibilidad de colaboración de los socios.

Asimismo, se encontró que Chen, Lin y Yen (2014) construyeron un modelo de intercambio de conocimientos a través de la cadena de suministro en el que factores como los objetivos compartidos, la integración social relacional y la estrategia de influencia se investigaron para determinar si actúan como fuerzas impulsoras principales para desarrollar confianza entre las organizaciones y los diversos miembros de la cadena de suministro. Para ello, realizaron una encuesta a 226 gerentes ubicados en los principales parques industriales de Taiwán; los resultados arrojaron que la confianza se impone cuando las organizaciones desarrollan metas compartidas, adoptan un modelo de integración social relacional e inician estrategias de influencia. Los autores señalan, además, que la confianza interinstitucional conduce a una mejor colaboración entre organizaciones y al intercambio de conocimientos. Los dos casos anteriores pueden explicar por qué en esta era de la economía de la red el intercambio de conocimientos entre organizaciones es una de las principales fuerzas impulsoras necesarias para racionalizar las actividades de la cadena de valor y maximizar los beneficios operacionales. El intercambio de conocimientos puede realizarse cuando los socios comerciales involucrados desarrollan con éxito la confianza y construyen asociaciones a largo plazo (Chen, Lin \& Yen, 2014).

\section{Modelos teóricos}

En el siguiente grupo se encuentran los modelos teóricos, representados por 21 artículos de la búsqueda que se tomaron en cuenta debido a que en gran cantidad de artículos se discuten planteamientos, propuestas o diseños teóricos del funcionamiento de la cadena de suministro que aún no han sido puestos en marcha, como es en el caso de los modelos prácticos, según se explica en el siguiente apartado. Para el grupo de modelos teóricos y de E-commerce (como se muestra más adelante), los resultados mostraron que, hoy en día, la mayoría de las empresas de tamaño medio empiezan a optimizar la coordinación de su cadena de suministro y la comunicación con sus clientes internos y externos mediante el uso de redes electrónicas, junto con las técnicas de comercio electrónico (Rehfeldt \& Turowski, 2000). Sin embargo, la gran mayoría de estas empresas no está agotando todo el potencial que los conceptos de soporte avanzado, como la coordinación y cooperación entre empresas y negocios, permiten. Por esta razón, los autores presentaron un enfoque basado en componentes más avanzados, como la adopción de tecnología activa, adaptable y automatizada de agentes para la coordinación (semiautomática) de las tareas empresariales y la distribución de información en todas las redes de producción, lo cual permitiría conceptos de repliegue y gestión del flujo de trabajo. Además, establecería las bases para una gestión del conocimiento exitosa.

Aunque estos conceptos han sido abordados durante algún tiempo por la comunidad científica, todavía no han encontrado su camino en la coordinación de negocios dentro de las redes de producción, razón por la cual Rehfeldt y Turowski (2000) se enfocaron en la manera de integrar algunos de estos conceptos en modelos de negocio de la próxima generación, caracterizados por la colaboración electrónica y la emergencia de los mercados electrónicos.

Por su parte, Bakas y Hoff (2010) afirman que el concepto de bienes inteligentes incluye tecnologías para identificar mercancías, y hacer seguimiento de estado y de lógica integrada. La arquitectura tecnológica de los bienes 
inteligentes es un campo emergente de investigación, pero la investigación sobre nuevos servicios potenciales y modelos de negocios para explotar los beneficios de los bienes inteligentes está mal representada. Los trabajos estudian cómo el concepto de bienes inteligentes puede permitir a los actores de la cadena de suministro innovar en sus modelos de negocio. En su estudio, Bekas y Hoff presentaron perspectivas de propuestas de valor únicas que pueden ser ofrecidas por los principales actores dentro del ámbito de transporte y logística: 1) usuarios de transporte; 2 ) proveedores de transporte; y 3) propietarios de infraestructura. Las implicaciones para los administradores se presentaron en forma de factores que permiten e inhiben las innovaciones de los modelos de negocio en las redes de transporte.

Por otra parte, Shamsuzzoha, Barros, Costa, Azevedo y Helo (2014) presentaron un modelo conceptual virtual, de referencia de fábrica, con el objetivo de brindar a las empresas orientaciones generales para gestionar y supervisar los procesos de negocio necesarios para crear, ejecutar y disolver una fábrica virtual. El modelo virtual de referencia de la fábrica se construyó teniendo en cuenta las necesidades de la industria y revisando la literatura en varios campos relevantes de investigación, tales como redes de colaboración, redes de suministro, redes de fabricación, gestión de la cadena de suministro y procesos empresariales.

La revisión también nos muestra que, con el advenimiento de la globalización y la correspondiente disminución en el número de barreras al comercio internacional, el comercio en la industria alimentaria abarcó las fronteras nacionales e internacionales. Con la creciente complejidad en las redes de la cadena de suministro es imperativo que todos los actores de la cadena de suministro de alimentos operen de una manera ética y sostenible. Jacob-John y Veerapa (2016) realizaron un documento que ilustra un modelo de proceso que reforzaría efectivamente los fundamentos éticos de una cadena de suministro de productos frescos.

Este trabajo presenta un modelo conceptual estructural que facilita la integración de la responsabilidad en las operaciones de las organizaciones dentro de una cadena de suministro de frutas y hortalizas. Este modelo explica la responsabilidad-centricidad a lo largo de toda la cadena de suministro, cuya implementación en el mundo empresarial práctico generaría un clima ético para la industria. Los autores postulan que esto influirá positivamente en las T de las cadenas de suministro de productos frescos: trazabilidad, testeabilidad, transparencia, confianza y tiempo.

\section{Modelos prácticos}

Los modelos prácticos que fueron agrupados en esta categoría ya se han aplicado en empresas manufactureras y de servicios y funcionan para las cadenas de suministros de diferentes industrias. En la búsqueda, este grupo está representados por veinte artículos, como se muestra en la Tabla 3.

Para el primer caso, empresas de manufactura, la búsqueda arrojó que las estrategias de gestión de inventario eficaces y su implementación eficiente se reconocen como elementos importantes en el modelo de negocio de un fabricante. Los objetivos son la reducción del nivel y el valor de los inventarios mantenidos, la alta velocidad en la transformación de los pedidos a los clientes y la flexibilidad para hacer frente a los cambios de la demanda (Chu \& Cheung, 2006). Por esta razón, dichos autores propusieron un modelo de negocio para la gestión de inventarios en una empresa de fabricación de productos plásticos de Hong Kong, con instalaciones de producción ubicadas en el continente. Una estrategia de cadena de suministro basada en la confianza mutua y la visibilidad de la información de inventario es esencial en la implementación de un modelo de negocio de este tipo. Se ha comprobado que el modelo es válido y factible. Se propone que el modelo también se puede aplicar con éxito para beneficios similares en otras empresas.

A medida que China surge como el centro de fabricación del mundo, más y más pequeñas y medianas empresas (pyme) comienzan a externalizar su producción y la gestión de la cadena de suministro en China. Pero es muy difícil administrar eficazmente la externalización internacional y la cadena de suministro, principalmente debido a su limitado tamaño y recursos. En este sentido, los agentes internacionales de outsourcing emergen como una solución potencial perseguida por muchas compañías occidentales. Actualmente, se tiene muy poca información tanto de aspectos prácticos como teóricos para comprender los agentes y sus características, razón por la cual Liu y Shi (2007) presentaron un documento basado en cuatro estudios de caso de agentes en tres sectores industriales importantes en China, el cual introduce modelos de negocio y rutas de crecimiento de los agentes internacionales de outsourcing de fabricación y establece un conjunto de indicadores de desempeño clave (KPI) para la evaluación. Los mencionados autores buscan ayudar a las pyme occidentales y a las empresas de alta tecnología a reconocer la maduración china y un sistema ideal para su implementación.

En los resultados de la búsqueda también se evidencia que, en los últimos dos años, altos directivos de varias compañías automotrices han comenzado a implementar un nuevo modelo de negocio llamado red de lealtad digital (DLN). El modelo permite a las empresas de cualquier industria recopilar y supervisar continuamente 
Sara Delgado Gil, Jairo Gonzalez Bueno, Jairo Núñez Rodríguez Modelo de negocios para la gestión de la cadena de suministro: una revisión y análisis bibliométrico

sus datos de clientes, productos y cadenas de suministro y, más precisamente, ajustar las actividades de ingeniería, producción, distribución y ventas / mercadeo para satisfacer la demanda actual y futura. Además, pueden utilizar los mismos datos para mejorar su asociación con los proveedores (Koudal \& Wellener, 2003).

Las redes de lealtad digital tienen tres componentes: (1) digital: las empresas utilizan tecnologías de información sofisticadas para administrar la información de manera más eficaz; (2) lealtad: el sistema está diseñado para orientar, satisfacer y retener a los clientes más rentables y, a su vez, utilizar información de clientes y datos de fidelización para hacer más eficiente la cadena de suministro; (3) redes: el sistema de información vincula proveedores, productores y clientes y se actualiza continuamente. Las compañías de DLN utilizan la tecnología de la información con recursos para aumentar la eficacia de la cadena de suministro y las iniciativas de gestión de relaciones con los clientes y desarrollan una sólida red de información digitalizada que vincula la cadena de valor y crea lealtad tanto en el comienzo como en el final de las operaciones comerciales.

Por el lado de la oferta, las compañías DLN monitorean continuamente el valor del cliente basándose en la retroalimentación sobre los requisitos del cliente, el historial de compras y las compras potenciales y confían en la tecnología digital para asegurarse de que sus clientes más valiosos se mantengan satisfechos. Hacen esto gestionando el inventario a través de la cadena de suministro para que los mejores clientes se sirvan en primer lugar, y lograr que la planificación de capacidad a corto y largo plazo responda a estas prioridades de los clientes.

Para el segundo caso, la industria de servicios, se encontró que Lukić et al. (2017) afirman que las tecnologías de red inteligente están trayendo innovaciones en las industrias de energía eléctrica, afectando a todas las partes de la cadena de suministro de electricidad y dando lugar a cambios en la estructura del mercado, modelos de negocio y servicios. Por tal motivo, los autores presentaron un modelo de negocios para una cadena de suministro de redes inteligentes.

El modelo se desarrolló para ofrecer a los mercados de electricidad los flujos de datos necesarios y la información importante para el proceso de toma de decisiones. El modelo propuesto brinda una manera de aprovechar eficazmente la nueva arquitectura de medición y las nuevas capacidades de la red para obtener el valor comercial inmediato de las enormes cantidades de datos dispares en las redes inteligentes emergentes. El modelo fue evaluado para el mercado serbio de electricidad en la empresa de transmisión de energía eléctrica Empresa

\section{Pública Elektromreža Srbije.}

Los resultados muestran que las soluciones de inteligencia empresarial pueden contribuir a una gestión más eficaz de las redes inteligentes, con el fin de asegurar que las empresas alcancen la sostenibilidad en los mercados de electricidad cada vez más competitivos a la vez que brindan servicios de alta calidad a los usuarios finales. Otro hallazgo importante de la búsqueda indica que la industria de la Unión Europea representa el 15\% del valor añadido europeo y el sector industrial desempeña un papel clave en la investigación, la innovación, la productividad, la creación de empleo y las exportaciones. Una medida importante para detener la declinación de la posición industrial global de Europa es representada por la industria 4.0 con el objetivo de aplicar la producción inteligente y la logística.

La industria 4.0 toca toda la cadena de suministro, que comprende el diseño y el desarrollo de productos, la gestión de operaciones y la logística y, al hacerlo, se requieren nuevos modelos y estructuras de negocio. Las evidencias empíricas de empresas exitosas revelan que los nuevos modelos de negocio están más orientados hacia el diseño de servicios, la innovación abierta y los enfoques de redes que en los conceptos tradicionales de las empresas industriales. En consecuencia, las empresas industriales tradicionales tienen que repensar y renovar sus estructuras y modelos de negocio en paralelo con la actual (Prause, 2015).

\section{E-commerce}

El grupo de e-commerce describe modelos de negocio para la gestión de las cadenas de suministro mediante el comercio electrónico. Aunque únicamente se encontraron nueve trabajos relacionados en esta categoría, son de gran importancia debido a que actualmente la mayoría de las empresas está optando por esta modalidad de negocio, principalmente por la accesibilidad del cliente a la empresa. Es por esto por lo que los grupos anteriores muestran gran relación con este. Con este argumento, los resultados de la búsqueda indican que el desarrollo en auge del Internet y el mundo de E-commerce han causado un enorme efecto en la conducta de los negocios globales en formas fundamentales. El comercio de alimentos frescos, una forma tradicional de proporcionar cosas para la vida cotidiana de la gente, se ha implicado enormemente en el entorno de Internet y el comercio electrónico. Para los alimentos frescos el comercio electrónico ha reducido las distancias, eliminando los intermediarios y favoreciendo la integración de su antigua cadena de suministro (Fan, Xu, Liang \& Guo, 2015). Dichos autores proponen una red de logística hacia adelante 
/ atrás y un modelo de planificación de rutas para empresas de comercio electrónico de alimentos frescos. Además, realizaron un cálculo en un caso de estudio de funcionamiento óptimo en un distrito de Shanghai, China, en empresas de comercio electrónico donde se utiliza para verificar la viabilidad del modelo mediante la adopción de algoritmo genético.

Li y Englert (2010), quienes afirman que como fase de desarrollo de E-commerce un negocio colaborativo necesita una poderosa plataforma de red para apoyar la compleja interacción y colaboración dentro o fuera de ella, presentaron un modelo de negocio colaborativo bajo el entorno del Internet y construyeron un marco conceptual paraunaplataformadenegociocolaborativobasadoen grid computing con la idea de SOA (arquitectura orientada a los servicios). SOA descubrió que puede existir colaboración entre los sistemas de $E$-commerce y los sistemas internos de las empresas. El marco conceptual también proporciona canales cooperativos para las empresas, socios, clientes y departamentos gubernamentales. Las interfases de los sistemas colaborativos están encapsuladas por el Servicio Grid y el E-commerce que se realiza mediante la búsqueda, integración e invocación del servicio de la red.

\section{Modelos business to business}

Finalmente, se encontraron cinco artículos sobre el grupo business to business (B2B), un modelo de negocio que se busca facilitar información entre las empresas con el fin de fortalecerlas con respecto a la información sobre los clientes. La relación entre las empresas puede ser horizontal o vertical.

Humphreys, Mclvor y Cadden (2006) proponen un modelo que tiene como propósito examinar cómo el comercio electrónico puede cambiar fundamentalmente los procesos interorganizacionales en la interfaz entre el comprador y el proveedor. Esto, mediante la identificación de una serie de factores que influyen en la interfaz comprador-proveedor en el comercio B2B. Este artículo lleva a la conclusión de que el comercio electrónico no solo permite el rediseño de los procesos organizativos internos, sino que se extiende tanto al comprador como al proveedor.

Por otro lado, Rabe y Weinaug (2016) presentan un modelo de procesos de negocio y herramientas de software basadas en ASP para permitir la integración sin problemas de servicios logísticos y servicios financieros en una red de manufactura B2B. Una parte importante de este proyecto es un estudio de campo que involucró a fabricantes y proveedores de servicios de muchos países europeos. El objetivo del estudio era ofrecer un modelo generalizado de los procesos en curso e identificar los potenciales económicos, así como las barreras en términos de disponibilidad de información, confianza, cambios de procesos requeridos, costos, etc.

\section{Comentarios}

Los resultados de la búsqueda arrojaron aportes valiosos para la investigación en curso en relación con los diferentes modelos de negocios para gestionar las cadenas de suministro empresarial, permitiendo hacer una clasificación de estos modelos en seis grupos: modelos genéricos, modelos de cadena de suministro, modelos teóricos, modelos prácticos, modelos e-commerce y modelos business to business (B2B).

Los modelos de negocio para la gestión de las cadenas de suministros tienen gran acogida por parte de los grupos de cadenas de suministros, modelos teóricos y modelos prácticos, pero sobre todo por los grupos de E-commerce y de B2B. Aunque los dos últimos no representan una mayoría en los resultados de la búsqueda, son de gran importancia actualmente para el funcionamiento efectivo y práctico de las cadenas de suministros.

Los indicadores bibliométricos fueron una herramienta útil para evaluar, determinar y proporcionar información sobre los resultados en el proceso investigativo que se realizó, permitiendo conocer la calidad de la actividad científica y la influencia tanto del trabajo como de las fuentes que se utilizaron.

En futuras investigaciones se pueden incluir el análisis de la ecuación de búsqueda en bases diferentes a Scopus y abarcar otros artículos asociados a la gestión de negocio y la cadena de suministro.

\section{Referencias}

Al-Debei, M. M., El-Haddadeh, R. \& Avison, D. (2008). Defining the business model in the new world of digital business: School of Information Systems, Computing and Mathematics.

Amit, R. \& Zott, C. (2001). Value creation in E-business. Strategic Management Journal, 22(6-7), 493-520. Recuperado de https://doi.org/10.1002/smj.187.

Andersson, B., Bergholtz, M., Edirisuriya, A., Ilayperuma, T., Johannesson, P., Gordijn, J., ... et al. (2006). Towards a reference ontology for business models. Conceptual Modeling-ER 2006.

Ayers, J. B. (2006). Handbook of supply chain management: CRC Press.

Bakas, O. \& Hoff, A. (2010). Business model innovations: The case of intelligent goods. Proceedings of APMS 2010 
Sara Delgado Gil, Jairo Gonzalez Bueno, Jairo Núñez Rodríguez Modelo de negocios para la gestión de la cadena de suministro: una revisión y análisis bibliométrico

- International Conference on Advances in Production Management Systems.

Ballou, R. H. (2004). Logística: Administración de la cadena de suministro: Pearson.

Blanchard, D. (2010). Supply chain management best practices: John Wiley \& Sons.

Camponovo, G. \& Pigneur, Y. (2003). Business Model Analysis Applied to Mobile Business. ICEIS (4).

Chen, Y. H., Lin, T. P. \& Yen, D. C. (2014). How to facilitate inter-organizational knowledge sharing: The impact of trust. Information and Management, 51(5). Recuperado de https://doi.org/10.1016/j.im.2014.03.007.

Chu, K. F. \& Cheung, C.-F. (2006). A manufacturing supply chain business model and its implementation in a plastics manufacturing company. Materials Science Forum (Vol. 532-533).

Day, R. A. (2005). Cómo escribiry publicar trabajos científicos: Pan American Health Org.

Díaz, R. A. (2014). Marketing y las nuevas tecnologías de información, aplicadas en los negocios. I+D Revista de Investigaciones, 4(2), 34-48.

Dubosson-Torbay, M., Osterwalder, A. \& Pigneur, Y. (2002). E-business model design, classification, and measurements. Thunderbird International Business Review, 44(1), 5-23. Recuperado de https://doi. org/10.1002/tie.1036

Elsevier. (2017). Scopus The largest database of peerreviewed literature. Recuperado de https://www. elsevier.com/solutions/ scopus.

Er, M. \& MacCarthy, B. (2006). Managing product variety in multinational corporation supply chains: $A$ simulation study. Journal of Manufacturing Technology Management, 17(8), 1117-1138.

Fan, S., Xu, X., Liang, C. \& Guo, J. (2015). Construction of Forward and Reverse Logistics Network and Route Planning of Fresh Food E-Commerce Enterprises. e-Business Engineering 12th International Conference (pp. 160-165). Recuperado de https://doi. org/10.1109/ICEBE.2015.35

Giannice, S. G. (2013). La logística y su esencia en los modelos de negocio. I congreso internacional sobre nuevas tendencias de la logística empresarial.

Hsieh, C.-S. \& Hung, J.-W. (2009). Integration agent negotiation and data global consistency forms automatic and none Bullwhip effect supply chain. WSEAS Transactions on Information Science and Applications, 6(6), 1037-1050.

Humphreys, P., Mclvor, R.\&Cadden,T.(2006). B2B commerce and its implications for the buyersupplier interface. Supply Chain Management, 11(2). Recuperado de https://doi.org/10.1108/13598540610652528.

Jacob-John, J. \& Veerapa, N. K. (2016). Integrating responsibility within food supply chains - A conceptual model. Acta Horticulturae (Vol. 1132). Recuperado de
https://doi.org/10.17660/ActaHortic.2016.1132.13

Kallio, J., Tinnilä, M. \& Tseng, A. (2006). An international comparison of operator-driven business models. Business Process Management Journal, 12(3), 281-298. Recuperado de https://doi.org/10.1108/14637150610667962

Koudal, P. \& Wellener, P. (2003). Digital loyalty networks: continuously connecting automakers with their customers and suppliers. Strategy \& Leadership, 31(6). Recuperado de https://doi.org/10.1108/10878570310505541

Leiden University. (2010). VOSviewer - Visualizing scientific landscapes [software].

Li,H.\&Englert, D. (2010). Conceptual framework for collaborative business based on service oriented grid computing. ICIME 2010-20102nd IEEE International Conference on Information Management and Engineering (Vol. 1). Recuperado de https://doi.org/10.1109/ICIME.2010.5478122

Lin,F.R.\&Shaw,M.J.(1998). Reengineeringtheorderfulfillment process in supply chain networks. International Journal of Flexible Manufacturing Systems, 10(3). Recuperado de https://doi.org/10.1023/A:1008069816606

Liu, Z. \& Shi, Y. (2007). The Emergence of International Outsourcing Agents in China: A New Type of Player in International Supply Networks. Journal of Asia Business Studies, 2(1). Recuperado de https://doi. org/10.1108/15587890780001278

Lukić, J., Radenković, M., Despotović-Zrakić, M., Labus, A. \& Bogdanović, Z. (2017). Supply chain intelligence for electricity markets: A smart grid perspective. Information Systems Frontiers, 19(1). Recuperado de https://doi.org/10.1007/s10796-015-9592-z

Magretta, J. (2002). Why business models matter. Recuperado de http://repository.binus.ac.id/2009-2/content/A0154/ A015481231.pdf

Manthou,V.,Vlachopoulou,M.\&Folinas, D. (2004).Virtual e-Chain (VeC) model for supply chain collaboration. International Journal ofProduction Economics, 87(3). Recuperado de https://doi.org/10.1016/S0925-5273(03)00218-4

Mentzer, J. T., DeWitt, W., Keebler, J. S., Min, S., Nix, N. W., Smith, C. D. \& Zacharia, Z. G. (2001). Defining supply chain management. Journal of Business Logistics, 22(2), 1-25.

Osterwalder, A., Pigneur, Y. \& Tucci, C. L. (2005). Clarifying business models: Origins, present, and future of the concept. Communications of the Association for Information Systems, 16(1), 1.

Panou, K. (2015). Assessing financial viability of the freemium model for supply chain service value networks: a case study of the container management service industry. International Journal of Integrated Supply Management, 9(3), 137-177.

Pinto, T. Y. A. \& Quintero, H. C. U. (2017). Modelo de negocios para comercialización de películas y recubrimientos comestibles en Bucaramanga. I+ D Revista de Investigaciones, 10(2), 15-25.

Prause, G. (2015). Sustainable business models and 
structures for industry 4.0. Journal of Security and Sustainability Issues, 5(2). Recuperado de https://doi. org/10.9770/ jssi.2015.5.2(3)

Rabe, M. \& Weinaug, H. (2016). Distributed analysis of financial and logistic services for manufacturing supply chains. International Technology Management Conference, ICE 2007.

Rajala, R. \& Westerlund, M. (2005). Business Models: A new perspective on knowledge-intensive services in the software industry: BLED 2005 Proceedings.

Rehfeldt,M.\&Turowski,K.(2000).Businessmodelsforcoordinating next generation enterprises. Proceedings - Academial Industry Working Conference on Research Challenges 2000: Next Generation Enterprises: Virtual Organizations and Mobile/Pervasive Technologies. Recuperado de https://doi. org/10.1109/AIWORC.2000.843288

Röglinger, M., Pöppelbu $\beta$ S. J. \& Becker, J. (2012). Maturity models in business process management. Business Process Management Journal, 18(2), 328- 346.

Seong Leem, C.,SikSuh,H.\&SeongKim, D. (2004).Aclassification of mobile business models and its applications. Industrial Management \& Data Systems, 104(1), 78-87. Recuperado de https://doi. org/10.1108/02635570410514115.

Shamsuzzoha, A., Barros, A., Costa, D., Azevedo, A. \& Helo, P. (2014). Conceptual reference model for virtual factory: Potentials for collaborative business. IFIP Advances in Information and Communication Technology (vol. 434).

Stähler, P. (2002). Business models as an unit of analysis for strategizing. Lausanne, Suiza: International workshop on business models.

Tan, K.-S., Ahmed, M. D. \& Sundaram, D. (2009). Sustainable warehouse management. Proceedings of the International Workshop on Enterprises \& Organizational Modeling and Simulation. Recuperado de http:// dl.acm.org/citation.cfm?id=1750415

The VantagePoint [software]. (2012): Search Technology. Recuperado de https://www.thevantagepoint.com

Tian-Min, C. (2009). Constructing Collaborative E-business Platform to Manage Supply Chain. International Conference on Information Management, Innovation Management and Industrial Engineering. Recuperado de https://doi.org/10.1109/ICIII.2009.255

Vurro, C., Russo, A., \& Perrini, F. (2009). Shaping sustainable value chains: Network determinants of supply chain governance models. Journal of Business Ethics, 90, 607-621.

\section{APÉNDICE A}

1. Lin, F.-R., \& Shaw, M. J. (1998). Reengineering the order fulfillment process in supply chain networks. International Journal of Flexible Manufacturing Systems, 10(3). https://doi.org/10.1023/A:1008069816606.

2. Manthou, V., Vlachopoulou, M., \& Folinas, D. (2004).Virtual e-Chain (VeC) model for supply chain collaboration.
International Journal of Production Economics, 87(3). https://doi.org/10.1016/\$0925-5273(03)00218-4

3. Wamba, S. F., \& Chatfield, A. T. (2009). A contingency model for creating value from RFID supply chain network projects in logistics and manufacturing environments. European Journal of Information Systems, 18(6). https:// doi.org/10.1057/ejis.2009.44

4. Cabral, I., Grilo, A., \& Cruz-Machado, V. (2012). A decisionmaking model for Lean, Agile, Resilient and Green supply chain management. International Journal of Production Research, 50(17). https://doi.org/10.108 0/00207543.2012.657970

5. Vurro, C., Russo, A., \& Perrini, F. (2009). Shaping Sustainable Value Chains: Network Determinants of Supply Chain Governance Models. Journal of Business Ethics, 90(SUPPL. 4). https://doi. org/10.1007/s10551010-0595-x

6. Muffatto, M., \& Payaro, A. (2004). Integration of webbased procurement and fulfillment: A comparison of case studies. International Journal of Information Management, 24(4). https://doi.org/10.1016/j. ijinfomgt.2004.04.008

7. Lefebvre, L., Lefebvre, É., Bendavid,Y.,Wamba, S., \& Boeck, H. (2005). The potential of RFID in warehousing activities in a retail industry supply chain. Journal on Chain and Network Science, 5(2). https://doi.org/10.3920/ JCNS2005.x059

8. Soroor, J., Tarokh, M. J., \& Shemshadi, A. (2009). Initiating a state of the art system for realtime supply chain coordination. European Journal of Operational Research, 196(2). https://doi.org/10.1016/j.ejor.2008.03.008

9. Humphreys, P., Mclvor, R., \& Cadden, T. (2006). B2B commerce and its implications for the buyer-supplier interface. Supply Chain Management, 11(2). https://doi. org/10.1108/13598540610652528 10

10. Sarkis, J., Meade, L. M., \& Presley, A. R. (2012). Incorporating sustainability into contractor evaluation and team formation in the built environment. Journal of Cleaner Production, 31. https://doi.org/10.1016/j. jclepro.2012.02.029

11. lakovou, E., Vlachos, D., \& Xanthopoulos, A. (2010). A stochastic inventory management model for a dual sourcing supply chain with disruptions. International Journal of Systems Science, 41(3). https:// doi. org/10.1080/00207720903326894

12. Merkuryeva, G., Merkuryev, Y., \& Vanmaele, H. (2011). Simulation-based planning and optimization in multiechelon supply chains. SIMULATION, 87(8). https://doi. org/10.1177/0037549710366265

13. Managing product variety in multinational corporation supply chains: A simulation study. Journal of Manufacturing Technology Management, 17(8). https:// doi.org/10.1108/17410380610707410

14. Chen, Y.-H., Lin, T.-P., \& Yen, D. C. (2014). How to facilitate 
Sara Delgado Gil, Jairo Gonzalez Bueno, Jairo Núñez Rodríguez Modelo de negocios para la gestión de la cadena de suministro: una revisión y análisis bibliométrico

inter-organizational knowledge sharing: The impact of trust. Information and Management, 51(5). https://doi. org/10.1016/j.im.2014.03.007

15. B2B integration in global supply chains: An identification of technical integration scenarios. Journal of Strategic Information Systems, 20(3). https://doi. org/10.1016/j. jsis.2011.04.001

16. Beheshti, H. M. (2010). A decision support system for improving performance of inventory management in a supply chain network. International Journal of Productivity and Performance Management, 59(5). https://doi.org/10.1108/17410401011052887

17. Svensson, G., \& Wagner, B. (2011). Transformative business sustainability: Multilayer model and network of e-footprint sources. European Business Review, 23(4). https://doi.org/10.1108/09555341111145735

18. Niesten, E., \& Alkemade, F. (2016). How is value created and captured in smart grids? A review of the literature and an analysis of pilot projects. Renewable and Sustainable Energy Reviews, 53. https://doi. org/10.1016/j.rser.2015.08.06

19. Liou, J. J. H., Tamošaitiene, J., Zavadskas, E. K., \& Tzeng, G.-H. (2016). New hybrid COPRAS-G MADM Model for improving and selecting suppliers in green supply chain management. International Journal of Production Research, 54(1). https://doi.org/10.1080/ 00207543.2015.1010747

20. Ilie-Zudor, E., \& Monostori, L. (2009). Agent-based framework for pre-contractual evaluation of participants in project-delivery supplychains. Assembly Automation, 29(2). https://doi. org/10.1108/01445150910945598

21. Decker, C., Berchtold, M., Chaves, L. W. F., Beigl, M., Roehr, D., Riedel, T., ... Herzig, D. (2008). Cost-benefit model for smart items in the supply chain. Lecture Notes in Computer Science (including subseries Lecture Notes in Artificial Intelligence and Lecture Notes in Bioinformatics) (Vol. 4952 LNCS). https://doi.org/10.1007/978-3-540-78731-0_10

22. Perrone, G., Bruccoleri, M., \& Renna, P. (2005). Designing and evaluating value added services in manufacturing e-market places. Designing and Evaluating Value Added Services in Manufacturing E-Market Places. https://doi. org/10.1007/1-4020-31521

23. Le, T. P. N., \& Lee, T.-R. (2013). Model selection with considering the $\mathrm{CO}<\mathrm{inf}>2</ \mathrm{inf}>$ emission alone the global supply chain. Journal of Intelligent Manufacturing, 24(4). https://doi.org/10.1007/s10845011-0613-6

24. Klobas, J. (1998). The virtual supply chain: A view of information flows, business structures and business opportunities. Business Information Review, 15(3). https://doi.org/10.1177/0266382984236867

25. Cadden, T., \& Downes, S. J. (2013). Developing a business process for product development. Business Process Management Journal, 19(4). https://doi. org/10.1108/ BPMJ-Jan-2012-0006
26. Hamilton, J., \& Selen, W. (2006). Service value encounters in the virtual service value chain:A conceptual framework. International Journal of Value Chain Management, 1(2). https://doi.org/10.1504/ IJVCM.2006.011182

27. Koudal, P., \& Wellener, P. (2003). Digital loyalty networks: continuously connecting automakers with their customers and suppliers. Strategy \&amp; Leadership, 31(6). https://doi.org/10.1108/10878570310505541

28. Ghobakhloo, M., Tang, S. H., Sabouri, M. S., \& Zulkifli, N. (2014).The impact of information system-enabled supply chain process integration on business performance: A resource-based analysis. International Journal of Information Technology and Decision Making, 13(5). https://doi.org/10.1142/ S0219622014500163

29. Addo-Tenkorang, R., \& Helo, P. T. (2014). ERP SaaS value chain: A proposed SaaS model for manufacturing SCM networked activities. International Journal of Business Information Systems, 17(3). https:// doi.org/10.1504/ IJBIS.2014.064980

30. Kristianto, Y., Ajmal, M. M., \& Helo, P. (2011). Advanced planning and scheduling with collaboration processes in agile supply and demand networks. Business Process Management Journal, 17(1). https:// doi. org/10.1108/14637151111105607

31. Macchion, L., Danese, P., \& Vinelli, A. (2015). Redefining supply network strategies to face changing environments. A study from the fashion and luxury industry. Operations Management Research, 8(1-2). https://doi.org/10.1007/s12063-014-0097

32. Cheng, T.M.(2009). Constructing collaborativeE-business platform to manage supply chain. In 2009 International Conference on Information Management, Innovation Management and Industrial Engineering, ICIII 2009 (Vol. 2). https://doi.org/10.1109/ ICIII.2009.255

33. Hsieh, C.-S., \& Hung, J.-W. (2009). Integration agent negotiation and data global consistency forms automatic and none bullwhip effect supply chain. WSEASTransactions on Information Science and Applications, 6(6).

34. Lukić, J., Radenković, M., Despotović-Zrakić, M., Labus, A., \& Bogdanović, Z. (2017). Supply chain intelligence for electricity markets: A smart grid perspective. Information Systems Frontiers, 19(1). https://doi.org/10.1007/s10796015-9592-z

35. Liao, Y., \& Marsillac, E. (2015). External knowledge acquisition and innovation: The role of supply chain network-oriented flexibility and organisational awareness. International Journal of Production Research, 53(18). https://doi.org/10.1080/0 0207543.2015.1008106

36. Prause, G. (2015). Sustainable business models and structures for industry 4.0. Journal of Security and Sustainability Issues, 5(2). https://doi.org/10.9770/ jssi.2015.5.2(3)

37. Fiorencio, L., Oliveira, F., Nunes, P., \& Hamacher, S. (2015). Investment planning in the petroleum downstream 
infrastructure. International Transactions in Operational Research, 22(2). https:// doi.org/10.1111/itor.12113

38. Dietrich, B., Ettl, M., Lederman, R. D., \& Petrik, M. (2012). Optimizing the end-to-end value chain through demand shaping and advanced customer analytics. Computer Aided Chemical Engineering (Vol. 31). https:// doi.org/10.1016/B978-0-444-59507-2.50002-0

39. Papagiannidis, S., Bourlakis, M., \& Li, F. (2008). E-fulfilling the e-supply chain of digital print. International Journal of Information Technology and Management, 7(1). https://doi.org/10.1504/IJTM.2008.015891

40. Liu, Z., \& Shi, Y. (2007). The Emergence of International Outsourcing Agents in China: A New Type of Player in International Supply Networks. Journal of Asia Business Studies, 2(1). https://doi. org/10.1108/15587890780001278

41. Chu, K.-F., \& Cheung, C.-F. (2006). A manufacturing supply chain business model and its implementation in a plastics manufacturing company. Materials Science Forum (Vol. 532-533).

42. Wibowo, A. A., \& Suryanegara, M. (2016). On developing the model of Smart Logistic Transport in Indonesia. In 2016 IEEE International Conference on Management of Innovation and Technology, ICMIT 2016. https://doi. org/10.1109/ICMIT.2016.7605015

43. Masvosvere, D. J. E., \& Venter, H. S. (2016). Using a standard approach to the design of next generation e-supply chain digital forensic readiness systems. SAIEE Africa Research Journal, 107(2).

44. Rabe, M., \& Weinaug, H. (2016). Distributed analysis of financial and logistic services for manufacturing supply chains. In 2007 IEEE International Technology Management Conference, ICE 2007.

45. Jacob-John, J., \& Veerapa, N. K. (2016). Integrating responsibility within food supply chains - A conceptual model. Acta Horticulturae (Vol. 1132). https://doi. org/10.17660/ActaHortic.2016.1132.13

46. Jia, F., Gao, R., Lamming, R., \&Wilding, R. (2016). Adaptation of supply management towards a hybrid culture: the case of a Japanese automaker. Supply Chain Management, 21(1). https://doi.org/10.1108/ SCM-01-2015-0009

47. Alayet, C., Lehoux, N., Lebel, L., \& Bouchard, M. (2016). Centralized supply chain planning model for multiple forest companies. INFOR, 54(3). https:// doi.org/10.1080 /03155986.2016.1197544

48. Fan, S., Xu, X., Liang, C., \& Guo, J. (2015). Construction of Forward and Reverse Logistics Network and Route Planning of Fresh Food E-Commerce Enterprises. In Proceedings - 12th IEEE International Conference on E-Business Engineering, ICEBE 2015. https://doi. org/10.1109/ICEBE.2015.35

49. Masvosvere, D. J. E., \& Venter, H. S. (2015). A model for the design of next generation e-supply chain digital forensic readiness tools. In 2015 Information Security for South Africa - Proceedings of the ISSA 2015 Conference. https:// doi.org/10.1109/ ISSA.2015.7335075

50. Sharma, S., \& Pai, S. S. (2015). Analysis of operating effectiveness of a cold chain model using Bayesian networks. Business Process Management Journal, 21(4). https://doi.org/10.1108/BPMJ-10-20140105

51. Xu, W., Yang, Z., \& Wang, X. (2015). A technical and business perspective on wireless sensor network for manufacturing execution system. Mathematical Problems in Engineering, 2015. https:// doi.org/10.1155/2015/267195

52. Panou, K. (2015). An innovative business model for marketing service value networks in the logistics and supply chain industry. International Journal of Integrated Supply Management, 9(4). https://doi. org/10.1504/ IJISM.2015.070512.

53. Alfaro, L. A., Le, T. M. H., Choi, H. R., \& Cho, M. J. (2015). Deployment Model of Big Data for Port Logistics. Information (Japan), 18(1).

54. Panou, K. (2015). Assessing financial viability of the freemium model for supply chain service value networks: A case study of the container management service industry. International Journal of Integrated Supply Management, 9(3). https://doi.org/10.1504/ IJSM.2015.068097

55. Gutierrez, K. J. G., Arguello, L. V. P., \& Jaimes, W. A. (2015). Restructuration of logistic networks: An industrial case study in Colombia. In IAMOT 2015 - 24th International Association for Management of Technology Conference: Technology, Innovation and Management for Sustainable Growth, Proceedings.

56. Teixeira, K. C., \& Borsato, M. (2015). A supporting model for the dynamic formation of supplier networks. In Advances in Transdisciplinary Engineering (Vol. 2). https://doi.org/10.3233/978-1-61499-544-9269

57.Ali,A.,Hawryszkiewyc, I.,\&Chen,J.(2014).Senvicesforknowledge sharing in dynamic business networks. In Proceedings of the Australian Software Engineering Conference, ASWEC. https:// doi. org/10.1109/ASWEC.2014.39

58. Shamsuzzoha, A., Barros, A., Costa, D., Azevedo, A., \& Helo, P. (2014). Conceptual reference model for virtual factory: Potentials for collaborative business. IFIP Advances in Information and Communication Technology (Vol. 434).

59. Park, Y., Hong, P., Sugie, R., \& Callaway, S. K. (2014). Dynamic network innovation in emerging markets: From supply chain to demand chain. In PICMET 2014 - Portland International Center for Management of Engineering and Technology, Proceedings: Infrastructure and Service Integration.

60. Elahi, B., \& Franchetti, M. (2014). A new optimization model for closed-loop supply chain networks. In 2014 IEEE International Technology Management Conference, ITMC 2014. https://doi.org/10.1109//TMC.2014.6918604

61. Chu, L. Y., Chen, X. Q., \& Dang, S. (2013). Design and implementation of supply chain integrated system based on internet of things. Applied Mechanics and Materials (Vol. 433-435). https://doi.org/10.4028/ www.scientific. 
net/AMM.433-435.2395

62.Güleş, H. K., Ergen,Z., \&Karabo囚a, K. (2013). A mixed integer linear programming model proposal for network design of a multi-phase, environmental and flexible closed loop supply chain. Mediterranean Journal of Social Sciences, 4(10). https://doi.org/10.5901/ mjss.2013.v4n10p586

63. Xiao-Ping, B., \& Xu, C.-M. (2013). Strategy research for the supply chain management based on value chain analysis in construction enterprises. Journal of Theoretical and Applied Information Technology, 51(1).

64. Singh-Derewa, C, \& La Regina, V. (2013). Caravan Cargo Autonomous RendezvousAndVelocity Adjustment/Navigation - Financial model for on orbit services. In Proceedings of the Intemational Astronautical Congress, IAC (Vol. 14).

65. Rahman, M., \& Rahman, A. (2012). Integrating lean, six sigma and logistics supports in a supply chain model. In 62nd IIE Annual Conference and Expo 2012.

66. Lu, D. W., \& Wang, M. S. (2012). Exploring the steel bar logistics value chain in China. Materials Science Forum (Vol. 697-698). https://doi.org/10.4028/ www.scientific. net/MSF.697-698.570

67. Graf, H.-C. (2012). Practical effects of vendor managed inventory (VMI) on used logistics technologies. In International Conference on Industrial Logistics, ICIL 2012 - Conference Proceedings.

68. Mucha, S. (2011). Economictrends spawn new EMS business models. Printed Circuit Design and Fab/ Circuits Assembly, 28(2).

69. Bakas, O., \& Hoff, A. (2010). Business model innovations: The case of intelligent goods. In Proceedings of APMS 2010 - International Conference on Advances in Production Management Systems.

70. Schellenberg, S., Mohais, A., Wagner, N., Ibrahimov, M., \& Michalewicz, Z. (2010). Optimising supply chain networks by means of a hybridised simulationbased approach. In 2010 IEEE World Congress on Computational Intelligence, WCCI 2010 - 2010 IEEE Congress on Evolutionary -Computation, CEC 2010. https://doi.org/10.1109/CEC.2010.5586096

71. Andreea, D., Adrian, C. L., Alexandru, S., \& Daniel, D. S. (2010). Multi-agents and GIS framework for collaborative supply chain management applications: Case study: Internet trade business network. In Proceedings - 9th RoEduNet IEEE International Conference, RoEduNet 2010.

72. Li, H., \& Englert, D. (2010). Conceptual framework for collaborative business based on service oriented grid computing. In ICIME 2010 - 2010 2nd IEEE International Conference on Information Management and Engineering (Vol. 1). https://doi. org/10.1109/ ICIME.2010.5478122

73.Tan, K.-S., Ahmed, M. D., \& Sundaram, D. (2009). Sustainable warehouse management. In EOMAS 2009- International Workshop on Enterprise and Organizational Modeling and Simulation, Held in Conjunction with CAiSE 2009. https://doi. org/10.1145/1750405.1750415

74. Xu, Y., \& Chen, W. (2009). A B3C E-business model for chain industry based on third-party payment. In Proceedings - International Conference on Management and Service Science, MASS 2009. https:// doi.org/10.1109/ ICMSS.2009.5305243

75. Huiping, C. (2009). Research on model of enterprise threedimensional information resources management under E-commerce environment. In Proceedings - International Conference on Management and Service Science, MASS 2009. https://doi. org/10.1109/ICMSS.2009.5303152

76. Proceedings - 23rd European Conference on Modelling and Simulation, ECMS 2009. (2009). In Proceedings - 23rd European Conference on Modelling and Simulation, ECMS 2009.

77. Zhang, Y. (2009). Enterprise supply chain management based on integration cooperative internet information. In Proceedings - 2009 International Forum on Information Technology and Applications, IFITA 2009 (Vol. 3). https:// doi.org/10.1109/IFITA.2009.258

78. Wu, B., \& Liu, Z.-Y. (2009). Value chain model of knowledge innovation in service supply chain. In Information Systems in the Changing Era: Theory and Practice Proceedings of the 11th International Conference on Informatics and Semiotics in Organisations, ICISO 2009.

79. Yan, J., \& Zhang, Y. (2007). Supply chain business process reengineering directed by the project. In Proceedings of the IEEE International Conference on Automation and Logistics, ICAL 2007. https://doi. org/10.1109/ ICAL.2007.4338945

80. Kim, S. J., Deng, G., Gupta, S. K. S., \& MurphyHoye, M. (2007). Intelligent networked containers for enhancing global supply chain security and enabling new commercial value. In 3rd IEEE/Create-Net International Conference on Communication System Software and Middleware, COMSWARE. https://doi. org/10.1109/COMSWA.2008.4554493

81. So, H. W. T., Gunasekaran, A., \& Chung, W. W. C. (2006). Last Mile fulfilment strategy for competitive advantage. International Journal of Logistics Systems and Management, 2(4).

82. Proceedings: International Conference on Next Generation WebServices Practices NWeSP 2005.(2005).InProceedings - International Conference on Next Generation Web Services Practices, NWeSP 2005 (Vol. 2005).

83. Chandrasekaran, R. S., \& Rockstroh, J. (2002). Cross industry innovation: As the auto industry moves from steel and glass to a silicon-based product, supplier relationships take center stage. SAE Technical Papers. https://doi.org/10.4271/2002-01-0328. 